\title{
Effect of band occupations in intermediate-band solar cells
}

\author{
César Tablero Crespo
}

\author{
Keywords: \\ Optical properties \\ Photovoltaic \\ Solar fuel \\ Semiconductors \\ Efficiency
}

\begin{abstract}
A B S T R A C T
Intermediate band semiconductors represent an alternative to increasing the efficiency of solar energy converters. However, the experimental results obtained so far do not reflect the expectations. Many factors are unable to explain it. In this work we have removed some of the approximations used for the efficiency evaluation taking into account the occupation of the bands, the inter-band absorption coefficients, and the optical thickness simultaneously. In many cases the results lower the expectations of the maximum efficiencies.
\end{abstract}

\section{Introduction}

An intermediate-band (IB) semiconductor (Fig. 1) can be obtained by introducing one or more intermediate bands (IBs) into a wide singlegap semiconductor, with the usual valence band (VB) and conduction band (CB). The IB semiconductor is a single absorbent material (multigap semiconductor with three bands) and not to several layers of different absorbent materials (single-gap semiconductor with two bands) with different optical thicknesses connected in series. Therefore the optical thickness affects all inter-band transitions within the multi-gap semiconductor. The IB solar cells are known as one of the third generation solar cells (Luque and Martí, 2003; Green, 2003; Würfel, 2005; Nelson, 2003). The maximum efficiency of the an IB solar cell is larger than that of a tandem cell with two layers (Green, 2003; Luque and Martí, 2003, 1997; Nelson, 2003; Würfel, 2005) ( 63\% versus 55\% with maximum sunlight concentration). The value $\sim 63 \%$ is found in this work in the favorable cases of occupation of the IB.

With selective contacts to the VB and $\mathrm{CB}$, holes and electrons respectively can be extracted to an external circuit. The contacts allow a selective exchange of carriers (electrons or holes). In conventional semiconductors is done by two different semiconductor-metal junctions. To extract electrons (holes) from the $\mathrm{CB}(\mathrm{VB})$ a junction is ohmic for the electrons (holes) and rectifier for the holes (electrons). This allows the flow of one type of carriers, avoiding or obstructing the flow of the other type of carriers. The semiconductor junction to extract electrons from the $\mathrm{CB}$ (holes from the $\mathrm{VB}$ ) is usually a $n^{+}$-type ( $p^{+}$-type) doped semiconductor.

If no carriers can be extracted from the IBs, i.e. the IB is electrically isolated, the photocurrents is increased with respect to the host singlegap semiconductor. This is because the optical $\mathrm{V} \rightarrow \mathrm{I}$ and $\mathrm{I} \rightarrow \mathrm{C}$ transitions generate additional carriers (holes in the VB and electrons in the
$\mathrm{CB}$ ), that add to those generated by the usual $\mathrm{V} \rightarrow \mathrm{C}$ optical transitions. In this way, the photons with less energy than the energy bandgap of the host single-gap semiconductor, which were lost, are used to generate additional carriers. As the IB is electrically isolated from the contacts, the photovoltage is controlled by the wide bandgap. Therefore an increase in the photocurrent with the preservation of the photovoltage increases the efficiency of this type of solar converter.

The IB could also open the possibility of a further radiationless mechanism $\mathrm{CB} \rightarrow \mathrm{IB} \rightarrow \mathrm{VB}$. But the behavior as centers of non-radiative recombination depends on whether the states are localized or delocalized making up an IB. These intermediate states can be inserted into the bandgap using impurities. This can lead, in some cases, to localized defect states at low concentration (impurity-doping) or an energy band at high doping concentration (impurity-alloying). For impurity-doping these localized defect states act as Shockley, Read and Hall non-radiative recombination centers (Hall, 1952; Shockley and Read, 1952). In this case the energy of an excited carrier is dissipated by multi-phonon emission (Lang and Henry, 1975). It reduces the efficiency of the solar cells. Nevertheless, for the impurity-alloying case, the states make up an IB with delocalized states and the negative non-radiative recombination effect is suppressed (Luque et al., 2006; Tablero, 2009, 2012a). In the latter case the impurity concentration must exceed the threshold set by the Mott's transition (Luque et al., 2006).

When evaluating the maximum efficiency of this type of device, several approximations are made (Brown and Green, 2002; Luque and Martí, 1997): the cell operates at $300 \mathrm{~K}$, carrier mobilities are infinite (no ohmic losses), non-overlapping absorption coefficients, etc. Additionally, for the transitions involving the IB to generate more carriers, the IB must be partially occupied: it must have empty states for the $\mathrm{V} \rightarrow$ I transition to take place, and full states for the $\mathrm{I} \rightarrow \mathrm{C}$ transition to take place. If not, the efficiency would decrease and the IB solar cell would behave as one single-gap solar cell. 
(a)

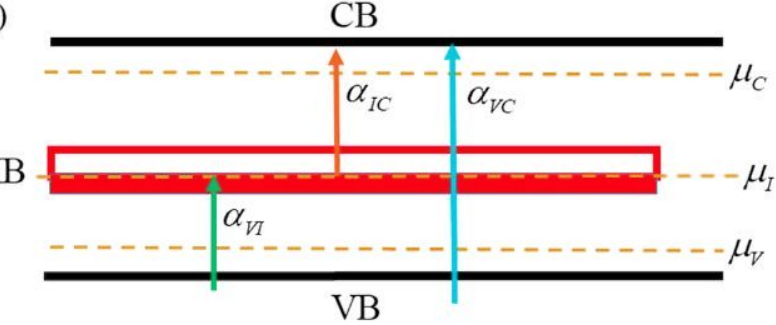

(b)

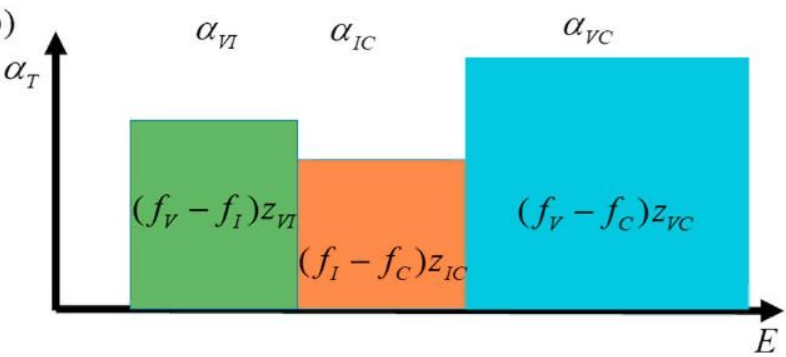

Fig. 1. Simplified (a) band diagram and (b) total $\left(\alpha_{T}\right)$ and inter-band $\alpha_{A B}$ absorption coefficients of an IB solar cell. $\mu_{A}$ and $f_{A}$ are the chemical potential and occupation factor associated with the A-band respectively. $z_{A B}$ depends on the inter-band $\mathrm{A} \rightarrow \mathrm{B}$ transition probability, and on the density of states of the $\mathrm{A}$ and $\mathrm{B}$ bands.

The experimental results related to the operation of IB solar cell (Ramiro et al., 2014) have not yet led to the expected efficiencies for this type of solar cell. Among many factors that could explain it would be the IB occupation, the weakness of the absorption coefficients, and the optical thickness of the samples.

Despite the importance of the IB occupation and its dependence on the optical thickness, not much attention has been paid to this fact. In this work we have carried out a study on the impact of these factors on the efficiency of the IB solar cell. Experimentally, based on the original IB solar cell model (Luque and Martí, 1997), the coefficient of absorption, thickness, and mainly the band occupation are almost never considered. For this reason, absence of experimental data regarding band occupation, it is very difficult to contrast with experimental data. However, the results of this work could justify why some IB solar cells do not improve the performance of traditional single-gap solar cells or do not reach the expected efficiency.

\section{Methodology}

The photocurrent $J$ resulting from $A$ to $B$ band transitions is the difference between the current due to the absorbed and emitted photons (Luque and Martí, 1997, 2003; Würfel, 2005; Green, 2003): $J_{A B}=J_{A B}^{(a)}-J_{A B}^{(e)}, \quad$ where $\quad J_{A B}^{(x)}=q \int a\left(E, \alpha_{T}, w\right)\left(\alpha_{A B} / \alpha_{T}\right) \phi_{\kappa}^{(A B)}(E) d E$ ( $\kappa=a, e$ for absorption and emission respectively). In this expression $\alpha_{A B}$ is the absorption coefficient for the $\mathrm{A} \rightarrow \mathrm{B}$ transitions, $\alpha_{T}$ is the total absorption coefficient $\left(\alpha_{T}=\sum_{A} \sum_{B>A} \alpha_{A B}\right), a\left(E, \alpha_{T}, w\right) \simeq 1-e^{-\zeta \alpha_{T} w}$ is the absorptivity $(\zeta$ is 1,2 both with and without the back reflector respectively), $w$ the thickness of the absorbent material, $q$ is the electron charge, and $\phi_{a}^{(A B)}$ and $\phi_{e}^{(A B)}$ are the incident and the emitted spectrum photon flux density respectively from the A to B band transitions. The $6000 \mathrm{~K}$ Black-body (BB) spectrum has been used as the incident spectrum $\left(\phi_{a}^{(A B)} \equiv \phi_{a}\right)$. Because of the distance from the sun's surface to the earth, at a point just outside the Earth's atmosphere the sun's surface power density $\left(62 \mathrm{MW} \mathrm{m}^{-2}\right)$ is reduced (on account of the reduced angular range of the sun) by 46,200 (Green, 2003; Nelson, 2003). It corresponds to the Black-body spectrum on earth without concentration, i.e. with a sunlight concentration factor $\mathrm{X}=1$. If the sunlight is concentrated then the incident spectrum will be multiplied by the sunlight concentration factor $X(1 \leq X \leq 46,200)$. Therefore, at maximum concentration
$\left(X_{\max }=46,200\right)$, the power density on earth is equal to that on the surface of a Black-body at $6000 \mathrm{~K}$. For the emitted spectrum we have used the standard approach considering that the device emits radiation as a generalized Black-body body (also known as Kirchhoff's and Planck's laws for non-black bodies (Green, 2003; Würfel, 2005)) at $\mathrm{T}=300 \mathrm{~K}$ (Luque and Martí, 1997, 2003; Würfel, 2005; Green, 2003) $\left(\phi_{e}^{(A B)}=E^{2}\left(2 \pi / h^{3} c^{2}\right)\left(\alpha_{A B} / \alpha_{T}\right) \sum_{P} \sum_{Q>P}\left(\alpha_{P Q} / \alpha_{T}\right) b_{P Q}\left(E, T, \mu_{Q P}\right) \quad\right.$ where $b_{B A}\left(E, T, \mu_{B A}\right)=\left[e^{\left(E-\mu_{B A}\right) / k T}-1\right]^{-1}$ is the Bose-Einstein factor, $k$ is the Boltzmann constant and $\mu_{B A}=\mu_{B}-\mu_{A}$ is the chemical potential associated with the radiation emitted between the $\mathrm{A}$ and $\mathrm{B}$ bands (Luque and Martí, 1997, 2003; Würfel, 2005; Green, 2003)). Note that the Kirchhoff's or Planck's law describes both the emission of thermal radiation for $\mu_{Q P}=\mu_{Q}-\mu_{P}=0$ and the emission of luminescence radiation for $\mu_{Q P} \neq 0$. With illumination, part of the electron population increase electrochemical potential energy because the $\mathrm{P} \rightarrow \mathrm{Q}$ transitions. Then the system develops inter-band chemical potentials $\mu_{Q P}$. As a consequence spontaneous emission is increased. The rate of emission depends upon $\mu_{O P}$ and the difference $\mu_{O P}$ of chemical potentials of the bands (or quasiFermi energies) is the chemical potential of the emitted photons.

The net current for the A band is $J_{A}=\sum_{B \neq A} J_{B A}$, with $J_{A B}=-J_{B A}$. For a IB semiconductor the carriers are extracted from the VB (hole current $J_{V}$ ) and CB (electron current $-J_{C}$ ), and no current is extracted from the IBs, i.e. $J_{I}=0(I \neq V, C)$. Therefore, the current density-voltage $(J-V)$ characteristic is obtained by solving the multi-variable nonlinear problem simultaneously where the set of equations are $J=J_{V}=-J_{C}, J_{I}=0(I \neq V, C)$, and the target magnitudes to be obtained are the chemical potentials $\mu_{A}$, where $V=\mu_{C}-\mu_{V}$. This photovoltage $V$ is lower than the energy gap $\left(E_{g}\right)$ of the absorbent semiconductor $\left(\mathrm{V}<E_{\mathrm{g}}\right)$. This is because part of the energy absorbed is reemitted by radiative recombination. Note also that the photon emission current depends on the voltage. In fact, for single-gap non-degenerate semiconductors with $\mathrm{VB}$ and $\mathrm{CB}$, the Bose-Einstein factor $b_{C V}$ of the emission current can be approximated by the classical Boltzmann statistics and then (Baruch et al., 1995; Green, 2003; Ross and Collins, 1980; Sze and $\mathrm{Ng}$, 2007) where $q V=\mu_{C V}$. Other recombination mechanisms (non-radiative, superficial, etc) also cause voltage losses and can be modeled with the current expression (Baruch et al., 1995; Ross and Collins, 1980; Sze and Ng, 2007) $J_{\text {loss }}(V) \approx J_{\text {loss }}(0) e^{q V / k T}$ if $q V \gg k T$.

Note that the both the absorption coefficient (via $\left(f_{A}-f_{B}\right)$, where $f_{A}$ are the Fermi-Dirac factors $\left.f_{A} \equiv f_{A}\left(E, T, \mu_{A}\right)=\left[e^{\left(E-\mu_{A}\right) / k T}+1\right]^{-1}\right)$ and the emission current (via the Boltzmann factors $b_{B A} \equiv b_{B A}\left(E, T, \mu_{B A}\right)=\left[e^{\left(E-\mu_{B A}\right) / k T}-1\right]^{-1}$, depend on the target variables $\mu_{A}$ of the multi-variable non-linear problem.

The energy conversion efficiency $\eta$ of a PVC is the relationship between the output power from the device $\left(P_{\text {out }}=J V\right)$ and the power from the incident (sun) spectrum $P_{\text {sun }}\left(\eta=P_{\text {out }} / P_{\text {sun }}\right)$. Therefore, in order to obtain the maximum efficiencies for an IB semiconductor it is necessary to solve a multi-variable non-linear optimization problem where the position of the IB within the energy bandgap is optimized simultaneously in order to obtain the maximum efficiency. For this the absorption coefficient is necessary.

The absorption coefficient depends on the occupation factors $f_{A}$, energies $E_{A}$, and density of states $g_{A}$ of the $A$-bands, and the square of the momentum matrix elements $p_{A B}$ between the $A$ and $B$ bands. If the band structure is isotropic then the absorption coefficient can be expressed as (Casey and Panish, 1978; Nelson, 2003):

$\alpha_{A B}(E) \sim(1 / E) \int\left[f_{A}(x)-f_{B}(x+E)\right]\left|p_{A B}\right|^{2} g_{A}(x) g_{B}(x+E) \cdot d x$

Usually in semiconductor physics and in solar cells the bands are considered isotropic (in most cases parabolic near the band edges). If the bands are not isotropic for the absorption coefficient should be used the general equation (Bassani, 1975):

$\alpha_{A B}(E) \sim(1 / E) \iint\left[f_{A}\left(E_{A}\right)-f_{B}\left(E_{B}\right)\right]\left|p_{A B}\right|^{2} \delta\left(E_{B}\left(\mathbf{k}_{B}\right)-E_{A}\left(\mathbf{k}_{A}\right)-E\right)$.

$d \mathbf{k}_{A} d \mathbf{k}_{B}$ 
where $\delta$ is the Dirac delta function and $\mathbf{k}$ represent points in the Brillouin zone, instead of Eq. (1) (Casey and Panish, 1978; Nelson, 2003). These equations can be applied to both bands and levels only considering that for $N_{A}$ degenerate levels with energy $E_{A}$ the density of states $g_{A}$ is proportional to the Dirac delta function: $g_{A}(x)=N_{A} \delta\left(x-E_{A}\right)$.

For low enough excitation levels, the absorption coefficients can be split approximately as $\alpha_{A B}(E) \sim\left(f_{A}-f_{B}\right) \cdot z_{A B}$, i.e. on a function depending on the band occupations $\left(f_{A}-f_{B}\right)$, often referred to as the band-filling factor (Haug, 2004), and on a term $z_{A B}$ depending on the transition probability and the density of states $\left(z_{A B}=\left|p_{A B}\right|^{2} g_{A}\left(E_{A}\right) g_{B}\left(E_{B}\right)\right)$.

\section{Results and discussion}

In most evaluations of maximum efficiencies the absorptivity, and therefore the absorption coefficient $\alpha_{A B}$, is considered as a step function (Luque and Martí, 2001; Nelson, 2003) with a value of not zero and constant only for energies above the energy bandgap $E_{A B}=E_{B}-E_{A}$, with $E_{B}>E_{A}$. The difference in occupation numbers $\left(f_{A}-f_{B}\right)$ is considered constant and equal to one, i.e. $\alpha_{A B} \equiv z_{A B}$. This consideration is equivalent to assuming that the bands are carrier reservoirs.

We will relax this approximation to take into account the dependence on the band occupation factors $f_{A}\left(0 \leqslant f_{A} \leqslant 1\right)$. To do so, as previously mentioned, the absorption coefficients are split into two terms, one dependent $\left(f_{A}-f_{B}\right)$ and the other independent $\left(z_{A B}\right)$ of the occupation factors, i.e. $\alpha_{A B}=\left(f_{A}-f_{B}\right) \cdot z_{A B}$. For $z_{A B}$ we use the conventional step functions: zero if the photon energy is lower than the energy bandgap between the bands $E_{A B}$, and a constant value $z_{A B}$ for $E_{A B} \geqslant 0$.

We consider a double-gap semiconductor with three bands, the usual VB and CB and, between them, an IB. In order to evaluate the dependence of the efficiencies on the IB occupation factor $f_{I}$ we consider the $\mathrm{VB}$ and the $\mathrm{CB}$, with occupation factors $f_{V}$ and $f_{C}$, almost filled and empty respectively $\left(f_{V} \sim 1\right.$ and $\left.f_{C} \sim 0\right)$, i.e. as electron and hole reservoirs respectively, and $z \equiv z_{V I}=z_{V C}=z_{I C}$.

In Fig. 2 the maximum efficiency is represented as a function of the energy band gap for several $p_{I}=f_{I} \cdot(z w)$ values. The set of values of $z w$, within the range $10^{+2} \leqslant z w \leqslant 10^{-1}$, include most of the possibilities for absorption coefficients and optical thicknesses of the solar cells. For example, considering an habitual average absorption coefficient (without taking into account the occupation factors) of $z=10^{+4} \mathrm{~cm}^{-1}$, then $10^{-2} \mathrm{~cm} \leqslant w \leqslant 10^{-5} \mathrm{~cm}\left(10^{+2} \mu \mathrm{m} \leqslant w \leqslant 10^{-1} \mu \mathrm{m}\right)$. In all cases: (i) when $p_{I}=f_{I}=0$ the results correspond to a single-gap semiconductor; (ii) for $z w \geqslant 10^{+2}$ the efficiencies are equal, because the absorptivity $a \sim 1$. The case $z w=10^{+2}$ is shown in panel a. The efficiencies for $z w>10^{+2}$ are equal to the results shown in panel a. Also, for this reason, the results when the occupation numbers are not considered for $z w \geqslant 10^{+2}$ (labeled as REF in figure) and $z w \rightarrow \infty$ (labeled as REF1 in figure) match. Therefore the quotient $\eta / a$ between the efficiency $\eta$ and the absorptivity $a$ is a constant that does not depend on $z w$ for $z w \geqslant 10^{+2}$.

In Fig. $2 \mathrm{~b} p_{I}=f_{I}$ because $z w=1$. In this case the values corresponding to $p_{I}$ and $\left(1-p_{I}\right)$ almost match. For example the results for the pairs of $p_{I}$ values $(0.0,1.0),(0.2,0.8)$, and $(0.4,0.6)$ are almost equal. The efficiencies are lower than those of the previous case $\left(z w \geqslant 10^{+2}\right.$, panel a), because the absorption capacity is lower. For the same reason the maximum efficiencies for single-gap semiconductors $\left(p_{I}=f_{I}=0\right)$ and IB semiconductor (curve labeled as 'REF1') are lower than when $z w \geqslant 10^{+2}$ (or when $z w \rightarrow \infty$, curve labeled as 'REF'). Finally when the absorbent capacity $z w$ is very poor, either because the absorption coefficient, the width, or both are very small, the efficiencies are very low (panel $\mathrm{c}$ in Fig. 2 for $z w=10^{-1}$ ).

In order to evaluate the influence on the absorption capacity $z w$ and on the occupation $f_{I}$ we have fixed the semiconductor gap to $E_{g}=E_{V C}=2 \mathrm{eV}$ next to the gap with maximum overall efficiency

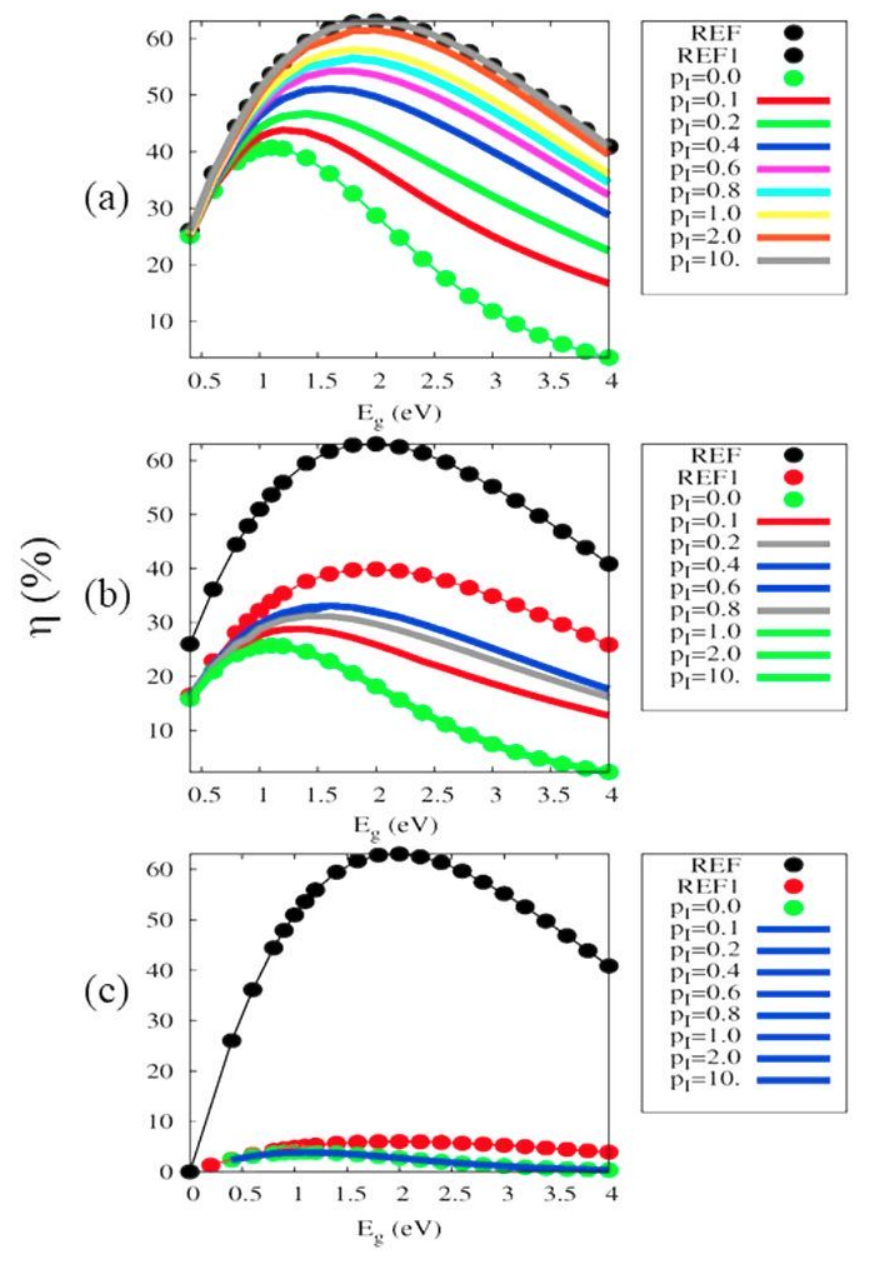

Fig. 2. Maximum efficiency as an energy gap function for several $p_{I}=f_{I} \cdot(z w)$ values using the $\mathrm{BB}$ spectrum, with maximum sunlight concentration $\left(X_{\max }=46,200\right)$, and with (a) $z w \geqslant 10^{+2}$, (b) $z w=10^{0}=1$, and (c) $z w=10^{-1}$. The curve labeled 'REF1' corresponds to the maximum efficiency when the occupation factors are not considered, i.e. $\left(f_{A}-f_{B}\right)=1$ for all transitions. The curve labeled 'REF' corresponds to the absolute maximum efficiency when $z w \rightarrow \infty$.

$(\sim 1.9 \mathrm{eV})$. The maximum efficiency, in accordance with the IB occupation for several values of $z w$, is represented in Fig. 3a. With the choice of the $x$ axis in the figure, the center corresponds to $f_{I}=1 / 2$. To the left $f_{I}$ decreases from $1 / 2$ to zero (empty IB), and to the right $f_{I}$ increases from $1 / 2$ to one (full IB). In all cases: (i) the maximum efficiencies extend to an interval of around $f_{I}=1 / 2$. This interval is larger when the absorption capacity $z w$ is greater. When $z w \rightarrow \infty$ the interval would include all possible values of $f_{I}$ occupation factors, except $f_{I}=0$ and $f_{I}=1$; (ii) only when $z w \geqslant 10^{+2}$, are the absolute maximum efficiencies (without considering the occupation factors and in the limit $z w \rightarrow \infty$ ) reached for $f_{I} \rightarrow 1 / 2$. In the scale of the figure, it seems that the maximum efficiencies are also reached when $z w=10^{+1}$. However, they are slightly different (63.06\% versus $62.86 \%$ for maximum sunlight concentration, and $45.22 \%$ versus $45.39 \%$ without sunlight concentration). This small difference cannot be appreciated in the scale of the figure; (iii) when the IB is completely full $\left(f_{I}=1\right)$ or empty $\left(f_{I}=0\right)$ (right- and left-hand limits of the horizontal axis in the figure respectively) the results coincide with the overall maximum efficiencies of a single-gap semiconductor with $E_{g}=2 \mathrm{eV}$.

The output voltage does not vary significantly in all cases. It is between $1.874 \mathrm{~V}$ and $1.879 \mathrm{~V}$. However, the IB position (Fig. 3b) varies considerably. For the limits $f_{I} \rightarrow 0$ (or $f_{I} \rightarrow 1$ ) the IB semiconductor behaves like a single-gap semiconductor. Then the IB approaches the 

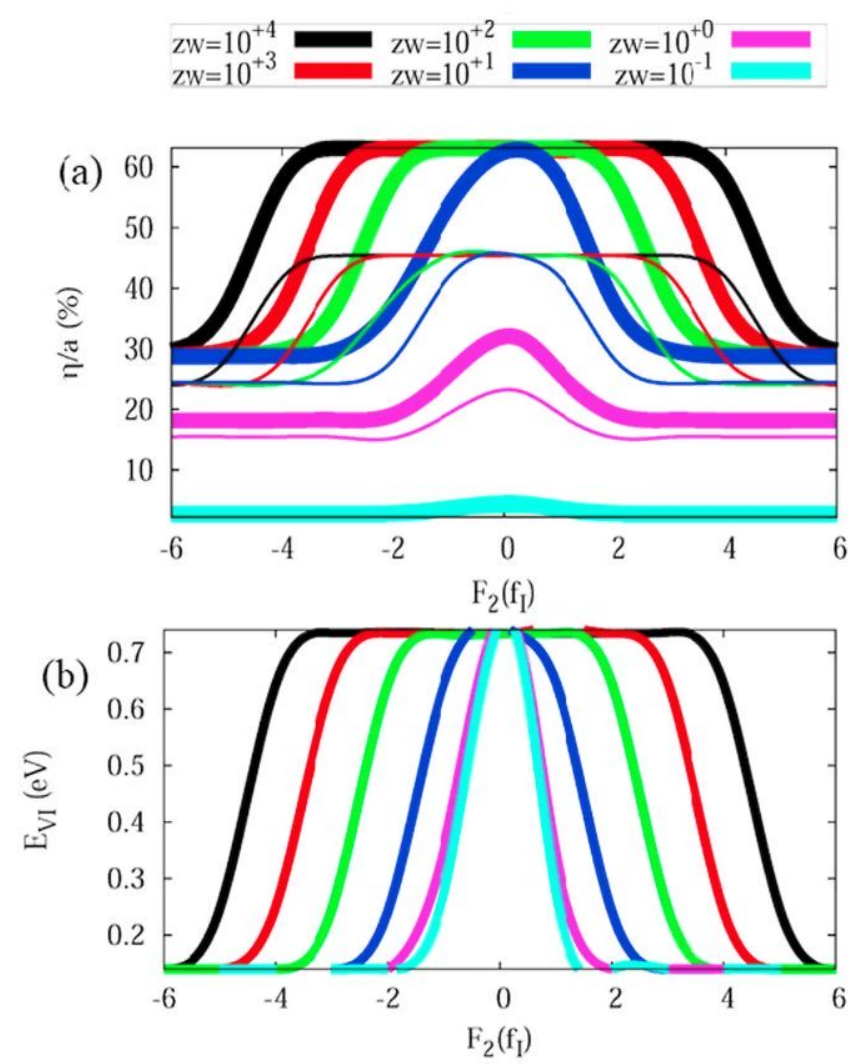

Fig. 3. (a) Maximum efficiency $\eta$, and (b) the energy position of the IB with respect to the $\operatorname{VB}\left(E_{V I}=E_{I}-E_{V}\right)$ as a function of the occupation factor $f_{I}$ for several $z w$ values of an IB semiconductor with an energy bandgap $E_{g}=E_{V C}=2.0 \mathrm{eV}$. The thin and thick curves correspond to the $\mathrm{BB}$ spectrum without concentration $(\mathrm{X}=1)$ and with maximum concentration $\left(X_{\max }=46,200\right)$ respectively. The function on the $x$-axe is: $F_{2}\left(f_{I}\right)=+\log \left(f_{I}\right)$ if $0 \leqslant f_{I}<1 / 2, F_{2}(1 / 2)=0$, and $F_{2}\left(f_{I}\right)=-\log \left(1-f_{I}\right)$ if $1 / 2<f_{I} \leqslant 1$. With this definition, the center of the figure corresponds to the IB full up to the middle, to the left the occupation decreases towards zero (empty IB), and to the right it increases towards one (full IB).

VB (or the CB). On the other hand, when $f_{I} \rightarrow 1 / 2$ and the absorption capacity $z w$ is large, the semiconductor efficiencies tend toward the maximum for an IB semiconductor.

So far we have obtained the overall maximum efficiencies by also optimizing the energy position of the IB in the bandgap (Fig. 3b). For this reason, when $f_{I} \rightarrow 0$ or 1 the IB tends towards the $\mathrm{VB}$ or $\mathrm{CB}$, and the IB semiconductor works like a single-gap semiconductor. The results could be very different if the IB energetic position is not optimized, i.e. it has a constant value for all $f_{I}$. Fig. 4 details the efficiency results for two IB semiconductors with $E_{V C}=2 \mathrm{eV}$ (Fig. 4a) and $E_{V C}=3 \mathrm{eV}$ (Fig. 4b) using the $\mathrm{BB}$ spectrum and $\mathrm{X}=1$. The first is very close to the optimal value. The second will illustrate better the latter conclusions. In this figure we have analyzed three different cases with respect to the IB position: (1) the position is optimized to reach the maximum efficiency for each value of $f_{I}$; the position is kept fixed (2) at the optimal value ( $E_{V I} \sim 0.7 \mathrm{eV}$ and 1.2 respectively) or (3) at a non-optimal value $\left(E_{V I}=0.9 \mathrm{eV}\right.$ and $1.0 \mathrm{eV}$ respectively). Cases 2 and 3 reflect the expected behavior of an IB semiconductor more accurately. Obviously, for case (3), with non-optimal $E_{V I}$, the efficiency is lower than in the other cases. Several other conclusions can be drawn: (i) the efficiencies for cases (1) and (2) are similar. There is a slight narrowing of the flat area of maximum efficiencies, and a decrease in the efficiencies in case (2) with respect to the optimum case (1) in the rest of the $f_{I}$ values. This confers certain strength to the optimal $E_{V I}$ values; (ii) in all cases by reducing or increasing the IB occupation $f_{I}$ with respect to the IB half-full, the
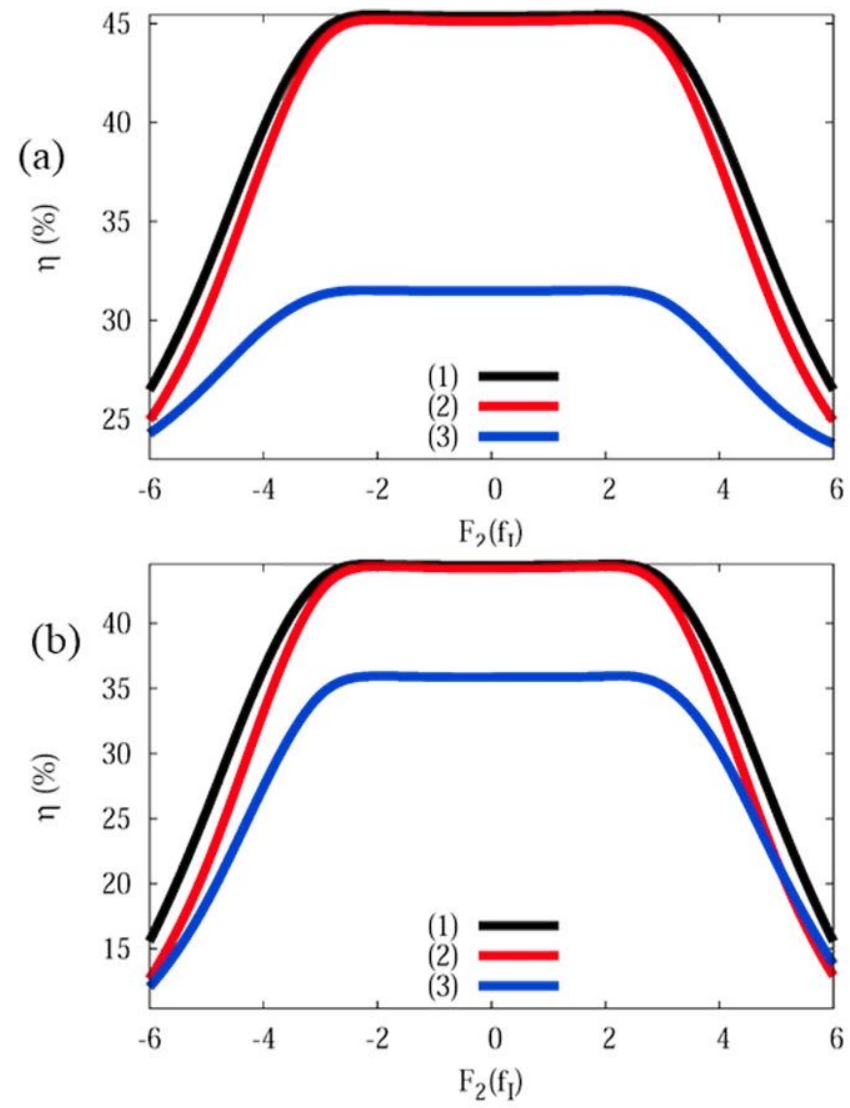

Fig. 4. Maximum efficiency $\eta$ as a function of the occupation factor $f_{I}$ for $z w=10^{+4}, \mathrm{X}=1$, and (a) $E_{V C}=2.0 \mathrm{eV}$ and (b) $E_{V C}=3 \mathrm{eV} . F_{2}\left(f_{I}\right)$ is defined in the caption of Fig. 2. The three curves in the figure correspond to different cases: (1) $E_{V I}$ is optimized for each $f_{I}$; (2) $E_{V I}$ is fixed to the optimum for $f_{I}=1 / 2$ ( $E_{V I} \sim 0.7 \mathrm{eV}$ and $1.2 \mathrm{eV}$ for $E_{V C}=2.0 \mathrm{eV}$ and $3.0 \mathrm{eV}$ respectively) for all $f_{I}$ values; and (3) $E_{V I}$ is fixed to non-optimal values $\left(E_{V I}=0.9 \mathrm{eV}\right.$ and $1.0 \mathrm{eV}$ for $E_{V C}=2.0 \mathrm{eV}$ and $3.0 \mathrm{eV}$ respectively) for all $f_{I}$ values.

efficiency tends towards that of a single-gap semiconductor with an energy bandgap $E_{V C}$ independently of the sub-bandgaps $E_{V I}$ and $E_{I C}$, i.e. of the position of the IB in the gap. This is reflected more clearly for $E_{V C}=3 \mathrm{eV}$ (Fig. $4 \mathrm{~b}$ ), where all the maximum efficiencies of singlegap solar cells for all sub-gaps (the optimal $E_{V I} \sim 1.2 \mathrm{eV}$ and $E_{I C} \sim 1.8 \mathrm{eV}$, and the non-optimal $E_{V I} \sim 1.0 \mathrm{eV}$ and $\left.E_{I C} \sim 2.0 \mathrm{eV}\right)$ are greater than $24 \%(30.7,27.2,28.8$ and $24.4 \%$ respectively), and larger than the $E_{V C}$ single-gap efficiency $(\eta \sim 10.6 \%)$. This fact, in principle, is non-intuitive. In the limits $f_{I} \rightarrow 0\left(f_{I} \rightarrow 1\right)$ we might think that the IB semiconductor behaves like a conventional single-gap semiconductor with a gap $E_{V I}\left(E_{I C}\right)$, and not with a gap $E_{V C}$. However this is because the contacts through which the carriers are extracted are to the VB and $\mathrm{CB}$. Therefore the IB net current must be zero and the voltage is determined by $E_{V C}$.

In this study we have taken $z \equiv z_{V I}=z_{V C}=z_{I C}$ in order to focus exclusively on the IB occupation. As $z_{A B}$ depends on the $\mathrm{A} \rightarrow \mathrm{B}$ transition probability and on the density of states of the A and B bands, it implicitly implies that: (i) the IB has a density of states similar to the VB and $\mathrm{CB}$. Then, as it is mentioned in the introduction, the non-radiative recombination is suppressed; (ii) all inter-band transitions have a similar probability but occurs at different rates because the absorption coefficients are different because they depends on the occupation: $\alpha_{V C}=z, \alpha_{V I}=\left(1-f_{I}\right) z$, and $\alpha_{I C}=f_{I} z$.

From the results shown in the figures the overall maximum efficiencies of the original IB solar cell model (Luque and Martí, 1997) are obtained approximately when $f_{I} \cdot(z w)>10$ with the constraint $0 \leqslant f_{I} \leqslant 1$. Else the efficiencies are lower than those predicted by 
the original model. However the efficiency of the ideal multi-gap solar cell is always larger than or equal to the efficiency of the ideal singlegap solar cell. Then the photovoltage is controlled by the wide bandgap $E_{V C}$ independently of the sub-bandgaps $E_{V I}$ and $E_{I C}$. Only in the unfavorable cases the efficiency is equal. For example when the IB is completely full or empty. Of course, this is true as long as the IB is well isolated from contacts to the $\mathrm{VB}$ and to the $\mathrm{CB}$. Otherwise, if the IB is not electrically isolated and one of the contacts is to the IB instead of to the VB or to the $\mathrm{CB}$, it would not be a multi-gap solar cell. This case would correspond to the usual single-gap solar cell where the voltage would be determined by one of the smallest bandgaps $\left(E_{I C}\right.$ or $E_{V I}$ respectively). Note also that the effect of absorption capacity $z w$ on efficiencies affects all solar cells, with single- or multi-gap semiconductors. If the absorptivity is less than unity, all solar cells absorb less photons, thus decreasing the efficiency.

As previously mentioned, it is difficult to contrast with experimental results. In some cases, due to the absence of occupation factors, optical thickness, and/or because the absorption coefficients (or other optical properties) obtained experimentally are not absolute values. They depend explicitly on occupations: $\alpha_{V I}=\left(1-f_{I}\right) z$, and $\alpha_{I C}=f_{I} z$. In the literature there are theoretical calculations of IB materials. In fact, this methodology to obtain efficiencies has previously been used with the absorption coefficients obtained from first-principles (Crespo, 2018; Tablero, 2016) as a photon energy function for the host semiconductor, and the habitual step absorption coefficients for modelling the $\mathrm{VB} \rightarrow \mathrm{IB}$ and IB $\rightarrow \mathrm{CB}$ transitions. In these works the optimal occupation $f_{I}=1$ / 2 is assumed, i.e. the effect of band occupations is not considered. Also some IB materials based on III-V, II-VI, and other semiconductors highly doped by a variety of transition elements have been described theoretically (Aguilera et al., 2010; Seminóvski et al., 2011; Tablero, 2012b, c; Tablero and Wahnón, 2003; Wahnón and Tablero, 2002). In most of these materials the IB occupation is $1 / 3$ or $2 / 3$. Therefore, as a consequence of the results in this work, they would be in the range of favorable $f_{I}$ cases (close to $f_{I}=1 / 2$ ) to obtain the maximum efficiencies when considering the band occupation and the usual thicknesses of solar cells.

Also, in the literature, there are some studies where optical thickness is considered. For example, according with results in Okada et al., 2015, the maximum efficiency is $52.8 \%$ for $w=500 \mathrm{~nm}$ InAs/GaAs QDIBSCs under maximum concentration when $z_{I C} / z_{V I}=0.2$ with $z_{I C} \geqslant 10^{5} \mathrm{~cm}^{-1}$. Although we have $z \equiv z_{V I}=z_{V C}=z_{I C}$ in order to focus exclusively on the IB occupation, the previous parameters would be equivalent to take in our model $w=500 \mathrm{~nm}, z=10^{5} \mathrm{~cm}^{-1}(z w=5)$ and $f_{I}=0.166$ (then $\alpha_{I C} / \alpha_{V I}=f_{I} /\left(1-f_{I}\right) \sim 0.2$ ). With this parameters the maximum efficiency is 52\%, similar to Okada et al., 2015.

\section{Conclusions}

We have carried out an evaluation of the variation of the main characteristics of an IB semiconductor according to the occupation of the IB and the optical thickness $w$, or more specifically with the absorption capacity $z w . z$ corresponds to the part of the absorption coefficient independent of the band occupation factors. The main conclusions can be summarized as: (1) when the absorption capacity $z w \geqslant 10^{+2}$ the efficiencies are equal because the absorptivity $a \sim 1 ;(2)$ the maximum efficiency is reached within an interval of around $f_{I}=1 / 2$ because the results for $f_{I}$ and $\left(1-f_{I}\right)$ almost match. This interval is larger, the bigger the absorption capacity $z w$. Furthermore, only when $z w \geqslant 10^{+2}$ are the absolute maximum efficiencies (limit $z w \rightarrow \infty$, and without considering the occupation factors) reached; (3) The results correspond to a single-gap semiconductor with energy bandgap $E_{V C}$ when $f_{I}=0$ or 1 regardless of whether the IB is located in the optimal energy position or not.
In addition to the IB occupation, the efficiency depends on $z$ which comes from the transition probability and the density of states. Therefore, to achieve high efficiencies, the IB density of states and the inter-band transition probabilities $\mathrm{VB} \rightarrow \mathrm{IB}$ and $\mathrm{IB} \rightarrow \mathrm{CB}$ must also be large.

\section{Acknowledgments}

This work has been supported by the MADRID-PV (S2013/MAE2780, FEDER FUNDS) and INVENTA-PV (TEC2015-64189-C3-1-R, MINECO/FEDER) National Spanish projects.

\section{References}

Aguilera, I., Palacios, P., Sánchez, K., Wahnón, P., 2010. Theoretical optoelectronic analysis of $\mathrm{MgIn}_{2} \mathrm{~S}_{4}$ and $\mathrm{CdIn}_{2} \mathrm{~S}_{4}$ thiospinels: effect of transition-metal substitution in intermediate-band formation. Phys. Rev. B 81, 075206.

Baruch, P., Vos, A.D., Landsberg, P.T., Parrott, J.E., 1995. On some thermodynamic aspects of photovoltaic solar energy conversion. Sol. Energy Mater. Sol. Cells 36, 201-222.

Bassani, G.F., 1975. Electronic States and Optical Transitions in Solids. Pergamon Press, Oxford, New York.

Brown, A.S., Green, M.A., 2002. Impurity photovoltaic effect: fundamental energy conversion efficiency limits. J. Appl. Phys. 92, 1329-1336.

Casey, H.C.J., Panish, M.B., 1978. Heterostructure Lasers: Part A, Fundamental Principles. Acad. Press, New York, NY.

Crespo, C.T., 2018. Potentiality of $\mathrm{CuFeO}_{2}$-delafossite as a solar energy converter. Sol. Energy 163, 162-166.

Green, M.A., 2003. Third Generation Photovoltaics. Advanced Solar Energy Conversion. Springer-Verlag, Berlin Heidelberg.

Hall, R.N., 1952. Electron-Hole recombination in germanium. Phys. Rev. 87 387-387.

Haug, H., 2004. Quantum Theory of the Optical and Electronic Properties of Semiconductors. World Scientific, Singapore River Edge, NJ.

Lang, D.V., Henry, C.H., 1975. Nonradiative recombination at deep levels in GaAs and GaP by lattice-relaxation multiphonon emission. Phys. Rev. Lett. 35, 1525.

Luque, A., Martí, A., 2003. Theoretical limits of photovoltaic conversion. In: Luque, A., Hegedus, S. (Eds.), Handbook of Photovoltaic Science and Engineering. John Wiley \& Sons Ltd.

Luque, A., Martí, A., 2001. A metallic intermediate band high efficiency solar cell. Prog. Photovolt. Res. Appl. 9, 73-86.

Luque, A., Martí, A., 1997. Increasing the efficiency of ideal solar cells by photon induced transitions at intermediate levels. Phys. Rev. Lett. 78, 5014-5017.

Luque, A., Martí, A., Antolín, E., Tablero, C., 2006. Intermediate bands versus levels in non-radiative recombination. Phys. B Condens. Matter 382, 320-327.

Nelson, J., 2003. The Physics of Solar Cells. Imperial College Press, London Reprint.

Okada, Y., Ekins-Daukes, N.J., Kita, T., Tamaki, R., Yoshida, M., Pusch, A., Hess, O., Phillips, C.C., Farrell, D.J., Yoshida, K., Ahsan, N., Shoji, Y., Sogabe, T., Guillemoles, J.-F., 2015. Intermediate band solar cells: recent progress and future directions. Appl, Phys. Rev. 2, 021302.

Ramiro, I., Martí, A., Antolín, E., Luque, A., 2014. Review of experimental results related to the operation of intermediate band solar cells. IEEE J. Photovolt. 4, 736-748.

Ross, R.T., Collins, J.M., 1980. Efficiency of quantum-utilizing solar energy converters in the presence of recombination losses. J. Appl. Phys. 51, 4504-4507.

Seminóvski, Y., Palacios, P., Wahnón, P., 2011. Intermediate band position modulated by $\mathrm{Zn}$ addition in Ti doped $\mathrm{CuGaS}_{2}$. Thin Solid Films 519, 7517-7521.

Shockley, W., Read, W.T., 1952. Statistics of the recombinations of holes and electrons. Phys. Rev. 87, 835-842.

Sze, S.M., Ng, K.K., 2007. Physics of Semiconductor Devices, third ed. Wiley-Interscience, Hoboken, NJ.

Tablero, C., 2016. Photovoltaic application of the multiferroic $\mathrm{Bi}_{2} \mathrm{FeCrO}_{6}$ double perovskite. Sol. Energy 137.

Tablero, C., 2012a. Ionization levels of doped copper indium sulfide chalcopyrites. J. Phys. Chem. A 116, 1390-1395.

Tablero, C., 2012b. Electronic and photon absorber properties of $\mathrm{Cr}$-doped $\mathrm{Cu}_{2} \mathrm{ZnSnS}_{4}$. J. Phys. Chem. C 116, 23224-23230.

Tablero, C., 2012c. Electronic, structural, and optical properties of the host and Cr-doped cadmium thioindate. J. Appl. Phys. 112.

Tablero, C., 2009. Effects of the impurity-impurity and impurity-host interactions on the charge density and the related processes. Phys. B Condens. Matter 404, 4023-4028.

Tablero, C., Wahnón, P., 2003. Analysis of metallic intermediate-band formation in photovoltaic materials. Appl. Phys. Lett. 82, 151-153.

Wahnón, P., Tablero, C., 2002. Ab initio electronic structure calculations for metallic intermediate band formation in photovoltaic materials. Phys. Rev. B - Condens. Matter Mater. Phys. 65, 1651151-16511510.

Würfel, P., 2005. Physics of Solar Cells. From Principles to New Concepts. WILEY-VCH Verlag GmbH \& Co, KGaA. 